\title{
BMJ Open Paediatric goal management training in patients with acquired brain injury: study protocol for a randomised controlled trial
}

\author{
Ruth Elizabeth Hypher, ${ }^{\oplus 1}$ Anne Elisabeth Brandt,, ${ }^{2,3}$ Kari Risnes, ${ }^{2,3}$ \\ Torstein Baade Rø, ${ }^{2,3}$ Eva Skovlund, ${ }^{4}$ Stein Andersson, ${ }^{5}$ \\ Torun Gangaune Finnanger, ${ }^{2}$ Jan Stubberud ${ }^{1,6}$
}

To cite: Hypher RE, Brandt AE, Risnes K, et al. Paediatric goal management training in patients with acquired brain injury: study protocol for a randomised controlled trial. BMJ Open 2019;9:e029273. doi:10.1136/ bmjopen-2019-029273

- Prepublication history for this paper is available online. To view these files, please visit the journal online (http://dx.doi. org/10.1136/bmjopen-2019029273).

Received 21 January 2019 Revised 27 June 2019 Accepted 08 July 2019

Check for updates

(C) Author(s) (or their employer(s)) 2019. Re-use permitted under CC BY-NC. No commercial re-use. See rights and permissions. Published by BMJ.

For numbered affiliations see end of article.

Correspondence to Dr Ruth Elizabeth Hypher; ruth_hypher@hotmail.com

\section{ABSTRACT}

Introduction Compromised integrity of the brain due to paediatric acquired brain injury $(\mathrm{pABI})$ has been associated with cognitive impairment, particularly executive dysfunction, in addition to somatic and emotional symptoms and reduced everyday function. Goal Management Training (GMT) is a cognitive rehabilitation intervention for improving executive function (EF) that has received empirical support in studies of adults with $A B I$. The purpose of the present study is to determine the efficacy of a recently developed paediatric version of GMT (pGMT) for children and adolescents with $\mathrm{AB}$ and reported executive dysfunction.

Methods and analysis This study protocol describes a parallel randomised controlled trial including allocation concealment and assessor blinding. Eighty survivors after $\mathrm{pABI}$, aged 10-17 years at the time of intervention, will be recruited. Participants will be randomly allocated to either pGMT $(n=40)$ or a psychoeducative control intervention ( $n=40$; paediatric Brain Health Workshop). Both interventions consist of seven group sessions for participants and parents, followed by external cueing and telephone counselling. The study also includes involvement of teachers. Assessments will be performed at baseline, immediately postintervention and at 6 months' follow-up. Primary outcome measure will be changes in daily life EF as reported by parents (The Behavior Rating Inventory of Executive Function). Secondary outcomes include other assessments of EF (neuropsychological tests and questionnaires). Furthermore, we aim to assess generalisation effects of pGMT on other cognitive functions, as well as emotional, behavioural, adaptive and family function, academic performance, fatigue and quality of life.

Ethics and dissemination Results from this study will be disseminated to relevant research, clinical, health service and patient communities through publications in peer-reviewed and popular science journals, in addition to presentations at scientific conferences. The study will be conducted in accordance with the Helsinki declaration and the Ethical Research Involving Children (ChildWatch International and Unicef). In accordance to Good Clinical Practice our study includes safety and quality monitoring guarantees in compliance with research ethics and safety. The trial will be reported in accordance with
Strength and limitations of this study

- Quality of design. A robust randomised controlled design, entailing an active control intervention, blinding of assessors, a well-defined primary outcome (with ecological validity) and a large sample size.

- Quality of intervention. Implementation of a theoretically based and manualised cognitive rehabilitation programme (Goal Management Training) already shown to be effective in adults, now adapted to children and enhanced by external cuing, counselling and involvement of parents and teachers.

- Outcome. Comprehensive outcome assessments including sustainability of effect (6 months follow-up).

- Participant heterogeneity may be regarded both as a limitation, with risk of effects in subsets of the study population being disregarded as null effects, and strength if results show effect across these differences (ie, generalisability).

- Another limitation is the risk of non-adherence to the interventions and loss of participants to follow-up due to comprehensive and time-consuming interventions.

the Consolidated Standards of Reporting Trials 2010 statement and Standard Protocol Items for Reporting in Trials recommendations, in addition to being registered at ClinicalTrials.gov. The study has been approved by the Regional Committees for Medical and Health Research Ethics Norway (2017/772).

Trial registration number NCT03215342.

\section{INTRODUCTION}

Acquired brain injury (ABI) is a diverse condition resulting from either traumatic brain injury (TBI) or non-traumatic injuries such as brain tumour, stroke, hypoxia or infections/inflammation to the brain. It is one of the leading causes of death and disability for children and adolescents. ${ }^{12}$ Although survival rates from paediatric $\mathrm{ABI}(\mathrm{pABI})$ is 
increasing with advances in prehospital care, surveillance and medical treatment, the possibility of poor outcome after survival remains. Indeed, pABI constitutes a major disruption to child development and may affect cognitive, behavioural, emotional, social as well as academic function. ${ }^{3-11}$ Previous assumptions that injuries early in life resulted in less adverse outcomes have been challenged by more recent research, suggesting that the young brain in fact may be vulnerable to more severe, diffuse and enduring deficits. ${ }^{12-15}$ Psychiatric disorders frequently follow pABI and occur at significantly higher rates than after orthopaedic injury without brain injury. ${ }^{16}$ The negative impact of pABI on social function and behaviour has been highlighted by several studies. ${ }^{341117}$ Further, fatigue is a problem encountered by a significant proportion of pABI patients and is associated with poor academic achievement, limited physical activity and socioemotional problems. ${ }^{18}{ }^{19}$ Moreover, poor postinjury family environment and resources have been shown to have negative effects on long-term social outcomes after early brain insult. ${ }^{15}$ Finally, following pABI, as many as $50 \%$ may experience persistent cognitive impairment, with a significant higher risk of executive dysfunction. ${ }^{892021}$

Executive dysfunction following pABI represents one of the most common and disturbing cognitive symptoms, impacting most domains of everyday life. ${ }^{92022-24}$ Executive function (EF) is a blanket term that embodies a collection of inter-related top down processes promoting the control and regulation of cognition (ie, goal-directed attention), emotion and behaviour. In particular, the domain of sustained attention is viewed as crucial to supporting the various $\mathrm{EF}$ processes, such as working memory, planning, monitoring, inhibiting and task switching. ${ }^{25}{ }^{26}$ Thus, executive processes play an essential role in a child's cognitive, behavioural, emotional and social function and development, with executive dysfunction potentially influencing the rehabilitation process, as well as postinjury academic achievement, social relationships, independence and future employment negatively. ${ }^{4927}$ Hence, interventions that target EF components, especially supporting the domain of sustained attention, may have the potential to significantly affect everyday function and may as such have life-long effects for children and adolescents with ABI.

As far as we know, there are no widely accepted standardised interventions targeting remediation of executive dysfunction for children and adolescents. ${ }^{28-30}$ In fact, cognitive rehabilitation after pABI varies excessively between region, hospital and community settings. ${ }^{31}{ }^{32}$ Effect studies of cognitive rehabilitation programme for children and adolescents are confounded with a multitude of challenges, including poor research design, divergent participant aetiologies, methodological inadequacies, lack of psychometrically sound and ecologically valid instruments for cognitive and behavioural assessments and finally inconsistency of outcome measures. ${ }^{28} 3133 \mathrm{In}$ a recent systematic review and metanalysis of cognitive interventions, only 13 randomised controlled studies were reported with only four including pABI populations, most of which involved computerised training. ${ }^{34}$ Sohlberg and colleagues ${ }^{29}$ identified five efficacy studies, ${ }^{35-39}$ evaluating attention training and metacognitive strategy training for children and adolescents with ABI. They conclude that while there is initial support for metacognitive interventions, the studies are not readily replicable as a number of clinical implementation factors were not properly identified.

Since the pABI is acquired during the course of development, this poses a particular challenge in research on this population. Thus, studies need to consider how developmental factors (eg, age at injury and intervention), maturity and awareness and access to early rehabilitation may impact the nature of rehabilitation and recovery. Furthermore, parent involvement in the intervention is highlighted by several studies as important for positive outcomes, especially for transfer to everyday life. ${ }^{3540}$ The school constitutes another significant learning arena in children's lives, essential for generalisation of intervention effects. ${ }^{40}$ To our knowledge, no previous studies have included parental and teacher components into a randomised controlled trial (RCT) to enhance EF in children.

Cognitive rehabilitation interventions that include metacognitive strategy training and problem-solving strategies have proved effective in treating EF impairments post-ABI in adults, with evidence of transfer to other domains such as quality of life (QoL) and emotional health. ${ }^{41}{ }^{42}$ One of the best validated interventions for executive dysfunction is Goal Management Training (GMT). ${ }^{25}$ 43-47 GMT has received empirical support in studies of adult patients with neurological conditions (eg, ABI) and in healthy elderly adults, ${ }^{45} 48$ with reports of improved sustained and executive attention ${ }^{434-51}$ and reduction of executive dysfunction in daily life. $^{44} 4652-55$ The theoretical foundation of GMT holds that the sustained attention system upholds higher order goals in mind while inhibiting automatic processes. ${ }^{25} 56$ Indeed, improved EF following interventions targeted at improving goal-directed attention regulation, such as GMT, is assumed to be a result of underlying alterations in brain networks supporting sustained attention. ${ }^{50} 5758$ The element of sustained attention is reinforced through mindfulness training. ${ }^{43} 59$ Furthermore, the participants are taught to be reflective with 'stop-and-think' strategies and thereby also raising awareness of attentional errors that often interfere with and obstruct task completion. Thus, the main objectives of GMT are to train participants to stop ongoing behaviour, to define goal hierarchies and monitor performance. ${ }^{43}$

As GMT has been shown to improve EF in various adult populations, application to broader patient- and age groups seems feasible. In fact, Krasny-Pacini and colleagues $^{60}$ investigated the efficacy of a multifaceted intervention programme with children with severe TBI $(n=5)$, including a paediatric GMT protocol. The study aimed at involving parents and teachers, ${ }^{61}{ }^{62}$ but 
unfortunately, involvement was low. ${ }^{60}$ Hence, the results of this pilot study were inconclusive, with only partial improvement of EF. The authors argue that the inconclusive results may be due to the inclusion of severe TBI and 'non-optimally functioning' families from low socioeconomic background. As GMT is a metacognitive intervention, it requires at least a moderate degree of awareness of deficits. ${ }^{25}$ As such, the paediatric version of GMT (pGMT) is probably not appropriate for patients with severe impairment (ie, severe TBI). Of, note, a preliminary version of the pGMT protocol to be used in the present study has been piloted on children with brain injuries $(n=13)$ with encouraging findings, suggesting enhanced attentional control and improved daily life $\mathrm{EF}$, as perceived by parents 6 months postintervention. ${ }^{63}$ In conclusion, there is great need for experimentally derived, prospective studies with randomly assigned experimental and control interventions, well-defined samples and investigator-masked outcome measures for children and adolescents with pABI. ${ }^{28}{ }^{62}$ It is critical that evidence-based cognitive remediation options are made available for this group. ${ }^{64}$ Given that heterogeneity is a hallmark characteristic of brain injury, larger multisite studies of systematic treatment are recommended to attain the volume and diversity necessary to discern the various factors that enhance intervention efficacy, as well as identify the specific, underlying mechanisms responsible for change. ${ }^{28} 2935$

The main objective of this RCT is to determine the efficacy of a modified version of GMT as a groupbased treatment programme for children and adolescents with pABI and EF deficits. Furthermore, we are interested in investigating potential transfer effects of pGMT to other central areas of functioning relating to emotional health, fatigue, social function and QoL. The present study aims to address several of the methodological shortcomings in previous studies by having a randomised controlled, multisite trial design, a relatively large sample size, blinded assessments, longterm follow-up, counselling of parents and teachers, in addition to external cueing to facilitate effective goal management in everyday life. The literature shows low correlation between neuropsychological tests and daily life (functional) EF. ${ }^{65}$ Thus, we have chosen a functional measure (The Behavior Rating Inventory of Executive Function (BRIEF) $)^{66}$ as a primary outcome, also based on prior research. ${ }^{55}$ The pGMT intervention will be compared with a psychoeducative control intervention (paediatric Brain Health Workshop (pBHW)), ${ }^{25}$ immediately after the intervention and at 6 months follow-up. The main study hypotheses are:

- Primary hypothesis: pGMT will result in a greater improvement in EF in daily life as reported by parents (BRIEF), compared with pBHW.

- Secondary hypothesis: pGMT will result in greater improvements in other EF domains (EF tests and functional $\mathrm{EF}$ ), as well as emotional, behavioural, adaptive and family function, academic performance, fatigue, QoL and other cognitive functions, compared with pBHW.

- Finally, we wish to examine whether there is an association between factors related to patient characteristics such as IQ, demographics and medical variables and treatment outcome.

\section{METHODS AND ANALYSIS}

\section{Study design}

The proposed study is a parallel RCT, comparing pGMT to an active control intervention (pBHW), using a repeated-measures design across three time-points (preintervention (T1), postintervention (T2) and 6-month follow-up (T3)) (figure 1).

The trial will be conducted and reported according to Consolidated Standards of Reporting Trials guidelines ${ }^{67}$ and Good Clinical Practice (GCP) ${ }^{68}$ The study flowchart is provided in figure 2 .

\section{Study setting}

Eighty participants with paediatric ABI will be invited to participate in the study based on hospital discharge diagnosis and record information. Interventions will be conducted at two sites in Norway-St Olav's Hospital $(\mathrm{SOH})$ in Trondheim and Oslo University Hospital, Rikshospitalet (OUH-RH). In addition, patients from the University Hospital of North Norway (UNN) will also be invited to participate. SOH, OUH-RH and UNN are the trauma referral centres for the Central, South-Eastern and Northern regions of Norway, respectively, and have a population base of more than two-thirds of the Norwegian paediatric population. Recruitment started in November 2017 and will continue until the summer of 2019.

\section{Population and eligibility criteria}

The study population will consist of children and adolescents diagnosed with ABI resulting from traumatic (TBI) and non-traumatic injuries (brain tumour, stroke, hypoxia/anoxia and brain infections/inflammations) from the age of 10 up to 17 years at the time of the intervention. Participation requires a period of at least 12 months since injury/illness or more than 12 months since ended cancer therapy, in addition to experiencing executive dysfunction in daily life as determined by a semistructured interview. Exclusion criteria are as follows: (1) injury acquired before 2 years of age; (2) cognitive, sensory, physical or language impairment affecting the capacity to attend regular school (ie, primarily follow educational goals of peers and regular classroom teaching) and/or complete the training programme; (3) preinjury neurological disease, severe psychiatric disorder and/or stimulant medication; (4) recently detected brain tumour relapse; (5) unfit for evaluation of outcome (independent evaluation by two investigators) and (6) not fluent in Norwegian.

\section{Sample size}

The proposed study aims at recruiting a total of 80 children and adolescents with pABI. Based on previous 


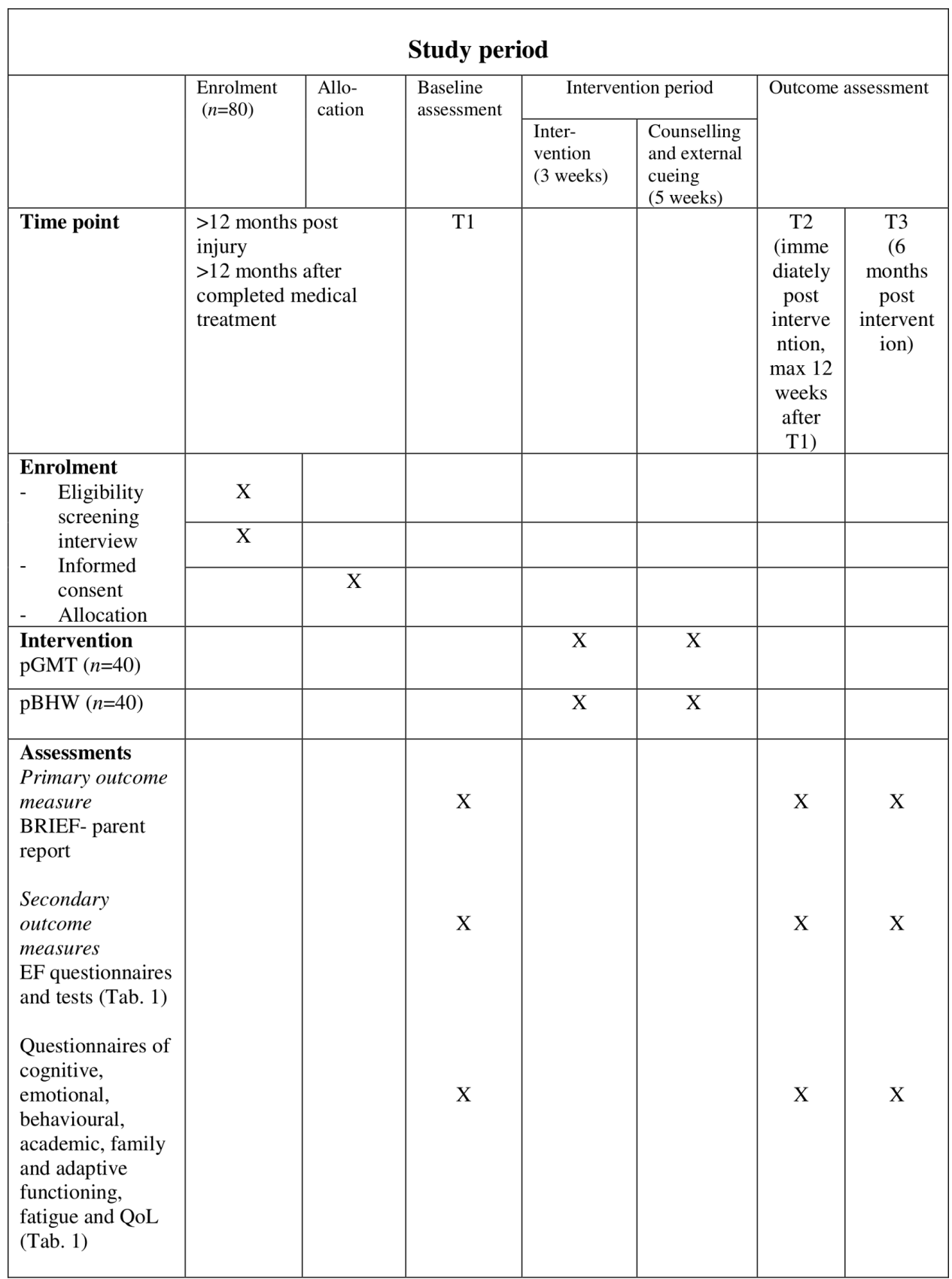

Figure 1 Study period illustrated by SPIRIT figure. BRIEF, Behavior Rating Inventory of Executive Functions; EF, executive function; pGMT, paediatric Goal Management Training; pBHW, paediatric Brain Health Workshop; SPIRIT, Standard Protocol Items for Reporting in Trials.

studies, the estimated annual incidence of TBI in Norway (aged $0-19$ years) is $1400 .{ }^{69}$ The prevalence of paediatric brain tumours in Norway is lower, with 40 new cases per year (survival rate is $80 \%),{ }^{70}$ with a high proportion of patients suffering from cognitive deficits. Incidence rates for childhood stroke vary from 1.3 per 100000 to 13.0 per $100000,{ }^{71}$ while rates of paediatric encephalitis-related hospitalisation range from 3 to 13 admissions per 100000 children per year (USA and Europe) ${ }^{72}$ Thus, the sample size is considered to be attainable, with reference to the total eligible population. Previous cognitive rehabilitation studies after pABI including metacognitive training have included $29,{ }^{61} 32,{ }^{73} 38^{39}$ and $161^{35}$ participants. As only one previous single-case study on pGMT in pABI exists to date,${ }^{60}$ this represents a challenge in estimating the required sample size in this proposed study. To document a clinically relevant effect as experienced by the child/adolescent and their family, we used the Global Executive Composite scale from the parental report on BRIEF $^{66}$ as the main outcome measure in the power analysis. Prior research on the effect of GMT in adults with $\mathrm{ABI}$ and spina bifida has reported moderate to large effect sizes. To detect an effect size of $d=0.7$ with a power $=0.80$ and $\alpha=0.05,32$ patients are needed in each group. 


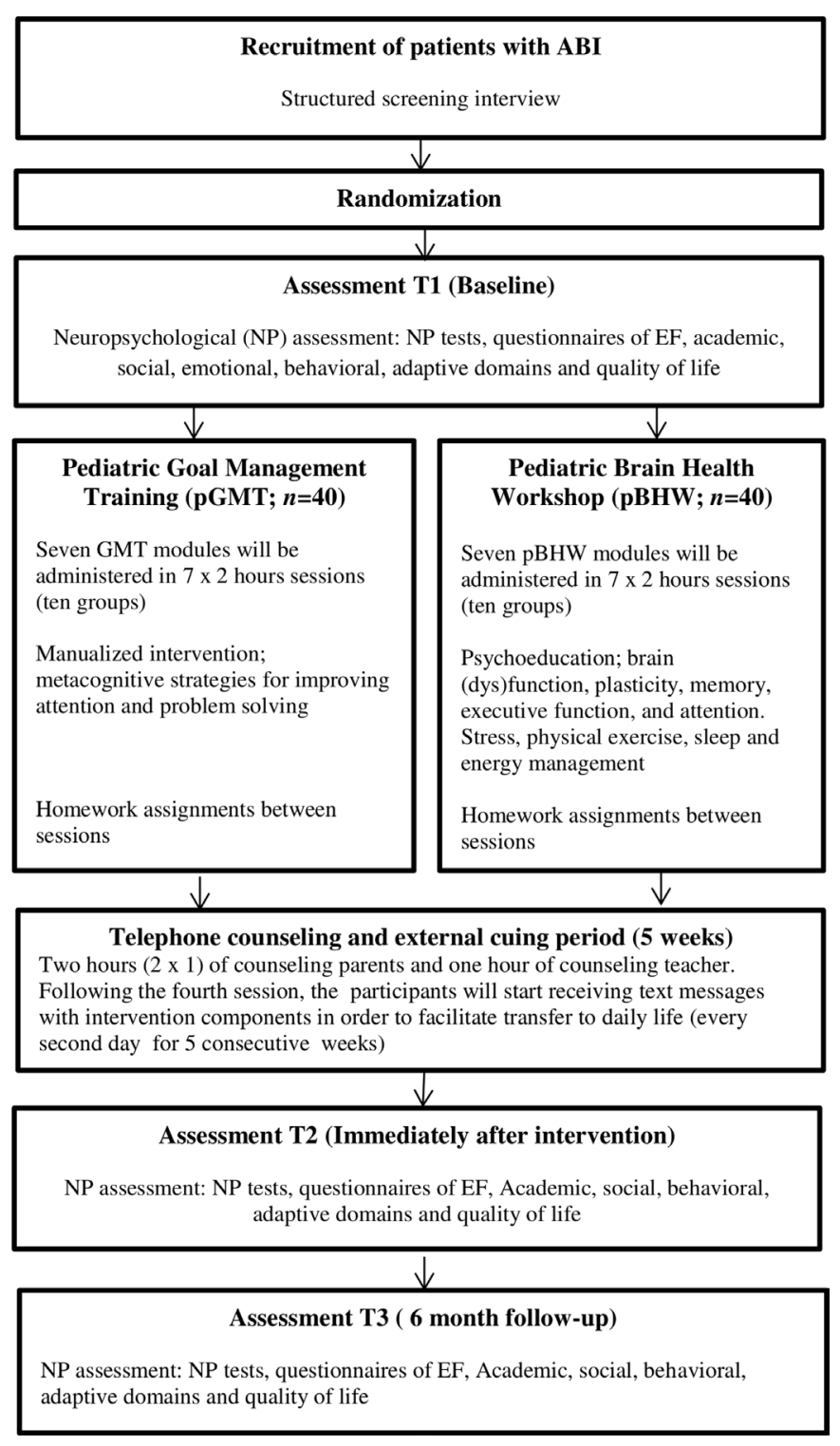

Figure 2 Flowchart for the study protocol. Cognitive Rehabilitation in paediatric $\mathrm{ABI}-$ a randomised controlled study. $A B I$, acquired brain injury; EF, executive function.

\section{Randomisation and blinding}

Participants will be randomised to either pGMT or pBWT in a 1:1 ratio, applying block randomisation and stratification by (1) research site (Trondheim or Oslo) and (2) age at the time of intervention (10-13 years or 14-17 years). The Unit for Applied Clinical Research, Norwegian University of Science and Technology will be responsible for the computerised randomisation but will not be involved in determination of participant eligibility, assessment or execution of the interventions. The block size is set to four due to the study design of the treatment. The block randomisation was set up in advance by the allocator and hidden from the executer to minimise the possibility of guessing the next allocation. Blinding will be applied to reduce systematic bias as a result of knowing the treatment allocations, with the following procedures: (1) Families and participants will not be informed about which intervention they have been assigned to (the term 'brain training' will be used consistently in both groups); (2) Test technicians who conduct baseline and follow-up assessments will be blinded to treatment allocation; (3) Therapists who administer the two interventions will be blinded to all test performance and evaluation; (4) The use of the Web Clinical Research Form (WebCRF) system, with data stored anonymously and only biostatistician and research assistant having access, minimises the likelihood of influence by the investigators. Furthermore, the interventions ( $\mathrm{pGMT}$ and $\mathrm{pBHW}$ ) will be conducted separately in time on both sites, thus reducing the chance of participants with differing allocations sharing information and experience. Additionally, participants will be explicitly asked not to discuss course content with test technicians assessing them or other potential participants outside of their group.

\section{Procedures}

Following a positive invitation response, written informed consent will be required from potential participants $(>16$ years) or primary caregivers (participants $<16$ years) before conducting a semistructured interview to determine EF symptoms and information of other inclusion/exclusion criteria that are not included in the hospital records. The interview section concerning daily EF consists of eight specific questions where the caregiver is asked to assess and describe the child's function, for example: 'Is it difficult for the child/adolescent to plan school assignments or leisure activities?'. Participants that have surpassed 16 years of age are invited to attend the interview. Successively, individuals that meet inclusion criteria will be designated a study number and randomly assigned to either of the two interventions by a computer-based algorithm in a webCRF. Once the participants have been randomised, a baseline assessment is completed on the first day of admission, prior to the first session of the group intervention, as illustrated in the flow chart in figure 2. Test technicians will receive training in test administration and scoring by a clinical neuropsychologist.

\section{Baseline variables}

At baseline (T1), the following information will be recorded in addition to tests included for primary and secondary outcome measures: age, sex, height, weight, medical status (medicines, sleep, appetite, sensory sensitivity), neurological status (cranial nerve, motor function, balance, sensibility), pain and hormone levels (thyroid-stimulating hormone, free thyroxine, adrenocorticotropic hormone, cortisol, insulin-like growth factor one hormone, luteinising hormone, follicle-stimulating hormone and serum osmolality), school attendance and special educational needs, as well as injury characteristics, age of injury and medical treatment. The demographic information will be collected in a standardised interview, medical examination (including neurological screening) by a physician and information in relation to injury/illness and treatment will be extracted from medical records. In 
addition, an optional blood test will provide hormonal level status. To provide an IQ estimate, the subscales vocabulary, similarities, digit span, coding, block design and matrix reasoning of the Wechsler Intelligence Scale for Children-Fifth Edition ${ }^{74}$ will be employed.

\section{Primary and secondary outcome measures}

The present study intends to investigate effects on a number of central domains pertaining to sequelae after pABI. Hence, a comprehensive assessment battery of common outcome measures has been selected (table 1). The primary outcome measure is the BRIEF, parent report. ${ }^{66}$ BRIEF is an 86 -item standardised questionnaire that captures parents perceptions of a child's EF in his or her everyday environment. Each item's frequency of occurrence is rated on a 3-point Likert scale from 1 (never) to 3 (often). It has demonstrated good reliability, with high test-retest reliability $(\mathrm{rs}=0.88$ for teachers, .82 for parents), internal consistency (Cronbach's $\alpha s=0.80$ 0.98 ), and moderate correlations have been detected between teacher and parent ratings $(\mathrm{rs}=0.32-0.34)$. The questionnaire has been applied to several clinical groups in Norway. ${ }^{75}$ Secondary outcome measures include: (1) domains specific to EF and (2) those related to other cognitive functions, as well as other functional areas such as emotional health, fatigue, social function and QoL (see table 1). Additionally, we have included measures of $\mathrm{EF}$ assumed to be more ecologically valid than traditional neuropsychological tests. Indeed, to better uncover challenges related to EF in daily activities we have translated and included a method that is designed as an ecological, open-ended assessment of EF in children, the 'Children's Cooking Task' (CCT). ${ }^{76}$ In addition to evaluating intervention satisfaction after each intervention week, intervention compliance will be assessed by modules completed by each child/adolescent.

\section{Interventions}

The intervention programme will be conducted in the same manner at both research sites according to preplanned standardised procedures. The two interventions are matched regarding non-specific factors (eg, structure, intensity, duration and therapist contact). Hence, the pGMT and pBHW protocols are group based and consist of seven modules of approximately 2-hour duration each (figure 3), both with standardised PowerPoint slides and participant workbooks. Between sessions, there will be homework assignments aiming to promote generalisation.

Two experienced clinical neuropsychologists will lead the group treatment sessions, each consisting of two to five participants, ideally four. The present study will aim at facilitating transfer from training to real life by including training and education of parents. A 1-hour review of the modules will be given to the parents by the same group therapists subsequent to each of the seven participant sessions, with the opportunity for questions and experience exchange. Following to the fourth session, the
pGMT participants will start receiving a text message stating 'STOP' (a key instruction in pGMT), while the pBHW will get a text message stating 'Brain training' every other day for five consecutive weeks until the end of the intervention period. This constitutes 18 text messages per participant and is included to cue goal management and encourage transfer to daily life. ${ }^{7879}$ The cueing time will be between 08:00 and 20:00 and will be changed routinely to prevent habituation. Two hours $(2 \times 1)$ of telephone counselling by one of the therapists, including both parent(s) and child when possible, will be conducted following the sessions during a 1-month period. The counselling mainly provides support to promote use of the techniques in everyday life by discussion of how the strategies (pGMT) or lifestyle advice and techniques (pBHW) may be implemented in home and school settings, in addition to clarifying possible obstacles and misunderstandings. Teachers will receive a 1-hour telephone counselling in the same period. The main purpose is to provide a brief review of the specific intervention strategy allocated and thus encourage teachers to consider how the strategies can be implemented in the school setting. In addition, the counselling may contribute to enhanced knowledge of pABI and cognitive dysfunction in general. Predetermined templates for the telephone counselling of parents/participants and teachers respectively ensures systematic and consistent procedures across sites, with specific protocol for each intervention.

Paediatric Goal Management Training and Paediatric Brain Health Workshop

In pGMT, participants are trained to use strategies such as stopping and orienting to relevant information, partitioning goals into subgoals, encoding and retaining goals, monitoring performance and mindfulness training. ${ }^{59}$ The training also uses discussion of participants' real-life attention deficits, in-session practice on tasks of attention supplemented with periodic alertness cueing and homework assignments. The manualised pGMT protocol is an adaptation of the adult version of GMT which has been translated into Norwegian. ${ }^{46} 4763$ The modifications of the GMT protocol for children included changes to make materials more child friendly and age appropriate (eg, examples and discussions more related to school and leisure activities). For example, 'The mental blackboard' (ie, working memory) became 'The brain blackboard', and 'The mental laboratory' (a way of thinking about, practising and experimenting with daily life skills) became 'The brain workshop'. Finally, the protocol has been compressed into seven modules (from 9). Following the pilot work by Stubberud and colleagues, ${ }^{63}$ the protocol has been further revised, primarily by simplifying language, reducing the amount of text, making examples more relevant to child and adolescent activities and adding more visual material. Nevertheless, all sessions will follow the same procedure; introduction to key concepts, practical exercises and discussion using examples from the participants' daily life. Homework assignments will include practical exercises and logging of activity and exercises in mindfulness. Similarly to the development of 


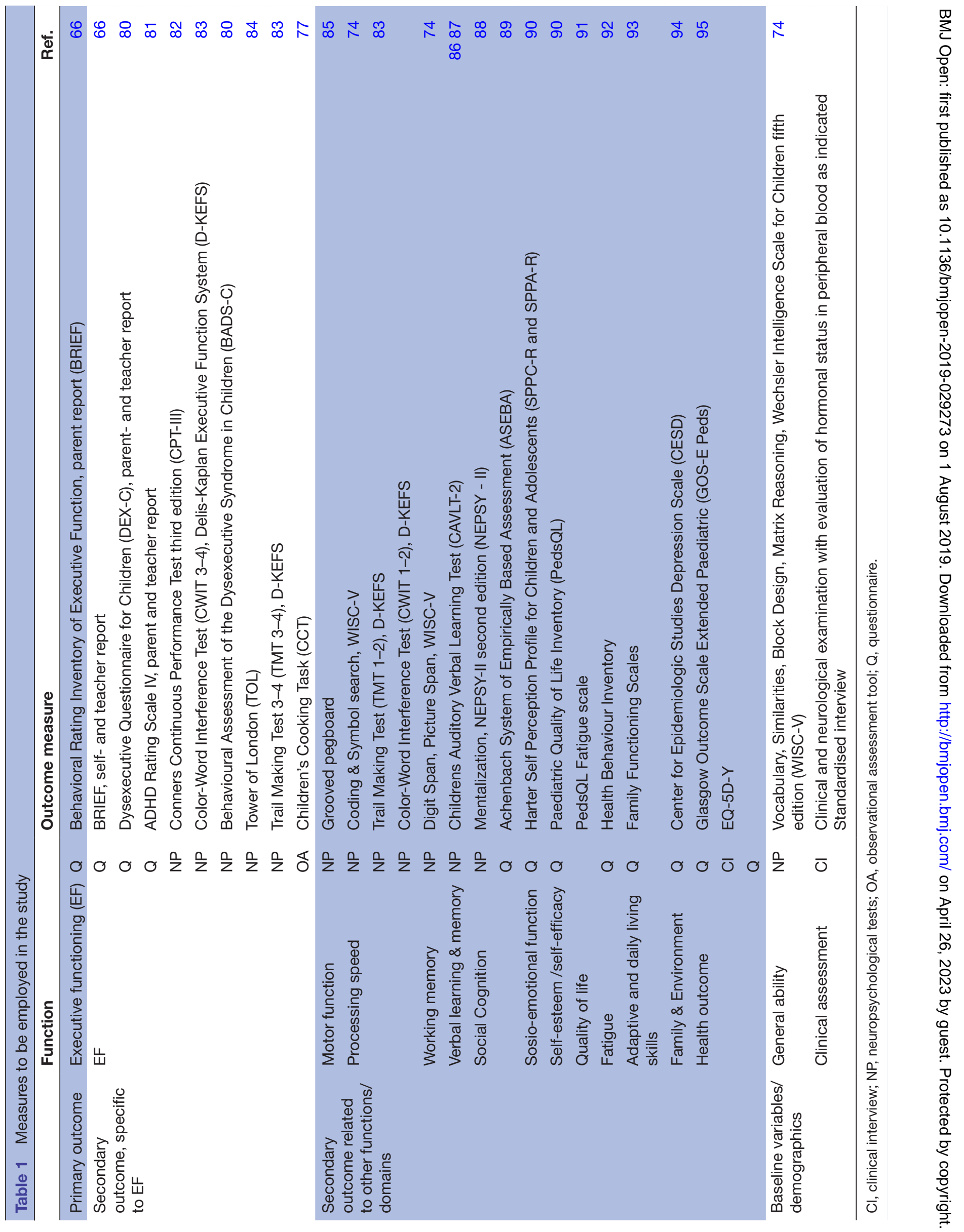




\begin{tabular}{|c|c|c|}
\hline $\begin{array}{l}\text { Paediatric Goal Management Training } \\
\text { sessions }\end{array}$ & $\begin{array}{l}\text { Paediatric Brain Health Workshop } \\
\text { sessions }\end{array}$ & $\begin{array}{l}\text { Inpatient } \\
\text { duration }\end{array}$ \\
\hline $\begin{array}{l}\text { Module 1: The absent mind, the present } \\
\text { mind } \\
\text { Module 2: Absentminded slip-ups }\end{array}$ & $\begin{array}{l}\text { Module 1: Introduction } \\
\text { Module 2: Neuroplasticity }\end{array}$ & 2 days \\
\hline \multicolumn{3}{|c|}{ Approximately 7 days at home including between session-assignments } \\
\hline $\begin{array}{l}\text { Module 3: The automatic pilot } \\
\text { Module 4: The brain blackboard }\end{array}$ & $\begin{array}{l}\text { Module 3: Memory I } \\
\text { Module 4: Memory II }\end{array}$ & 2 days \\
\hline \multicolumn{3}{|c|}{ Approximately 7 days at home including between session-assignments } \\
\hline $\begin{array}{l}\text { Module 5: State your goal } \\
\text { Module 6: Splitting tasks into subtasks } \\
\text { Module 7: Checking (Stop!) }\end{array}$ & $\begin{array}{l}\text { Module 5: Executive functioning and } \\
\text { attention } \\
\text { Module 6: Lifestyle and neuroplasticity I } \\
\text { Module 7: Lifestyle and neuroplasticity II }\end{array}$ & 3 days \\
\hline \multicolumn{3}{|c|}{$\begin{array}{l}\text { Two hours ( } 2 \times 1) \text { of counselling (parents + child when possible) following the intervention during a } 1 \\
\text { month period }\end{array}$} \\
\hline One hour of counselli & teacher in the same period for each child & \\
\hline
\end{tabular}

Figure 3 Description of the paediatric Goal Management Training and paediatric Brain Health Workshop interventions.

the pGMT, the pBHW is an adaptation of the adult version of BHW which has been translated into Norwegian. ${ }^{55}$ It comprises educational materials addressing brain injury and (dys)function, plasticity, memory and learning, executive function, fatigue and lifestyle interventions (eg, the effect of stress, sleep, exercise and nutrition on brain function) (figure 3). Homework and within-session activities will include sharing of the participant's experiences with the intervention topics in home and school settings, brain games, practical exercises and lifestyle advice, in addition to quizzes testing their acquired knowledge. Furthermore, the participants and parents are invited to discuss how the techniques and advice may be incorporated into their everyday routine.

\section{Statistical analysis}

Data analysis will follow the intention-to-treat principle and will be specified in a separate Statistical Analysis Plan. The analysis populations will be defined according to the following strategy: (1) The primary analysis population (modified intention-to-treat) will include all randomised participants who have completed baseline assessments. (2) The per protocol population will include all participants who have missed a maximum of two out of the seven group sessions. Results from all primary and secondary outcome measures will be presented separately by treatment allocation. A description of participant characteristics and baseline measurements will take place when all groups have carried through the baseline assessment (T1). The final analysis of the RCT will be conducted following the 6-month (T3) follow-up for participants, anticipated to be December 2019. For continuous outcomes, results will be presented as means and SD of age-corrected standardised scores defined from normative samples. The primary analyses will be performed by a linear mixed model with time point, intervention and the interaction between intervention and time as fixed factors. Supplementary analyses will be performed using linear regression. Sensitivity analyses will include adjustment for baseline factors where there is an obvious imbalance between the two groups. For binary outcomes, results will be presented as the number and proportion with impairments for each of the outcomes presented separately by treatment groups. Comparisons between groups will be done by $\chi^{2}$ tests. Additional sensitivity analyses adjusting for any imbalanced baseline factors will be performed by multiple logistic regression models. ${ }^{28} 29$ Significance level is set to $p \leq 0.05$. Due to the issues of multiple testing in assessment of a number of secondary outcomes, results will be interpreted according to magnitude of the group difference (effect size) rather than relying solely on significance levels.

\section{Ethics and dissemination}

Data management and monitoring

The study is set up to meet $\mathrm{GCP}^{68}$ quality and safety standard. A secure, web-based system (WebCRF) will be used to ensure storage of sensitive information and management of data files. The system ensures quality of data entry and allows for continuous monitoring of the study, as well as enabling download of data files for statistical analyses. Furthermore, participants will be allocated a unique research identification number prior to assessment. Consent form and documents containing personal information will be kept in a locked filing cabinet in a locked office within the hospital. A list of names and corresponding identification numbers will be kept separately and securely in the locked filing cabinet as well as on a password-protected hospital server. An external monitor agreement includes monitoring of protocol adherence, consent, randomisation and assessment of data quality during the study period. 


\section{Ethics and safety aspects}

The aims and methods in this project are not contrary to legislation, norms or values within the Norwegian society. Lastly, the trial will be reported in accordance with the CONSORT 2010 statement, ${ }^{67}$ in addition to being registered at ClinicalTrials.gov.

\section{Dissemination and user involvement}

Research findings from the described study will be disseminated through peer-reviewed research journals, conferences and patient organisations.

\section{Patient and public involvement}

Development of cognitive rehabilitation is highly requested by user organisations. We have identified several potential users; patients, their families, patient organisations and municipality services. This study seeks user involvement in all project phases. User involvement was included in the planning process through meetings and interviews with previous patients with pABI, patient organisations and municipality services, as well as execution of pilot studies. As such, the development of the study design, research questions and choice of outcome measures have been informed by patients' priorities, experience and preferences. The patients will not, however, be involved in the recruitment to and conduct of the study. Furthermore, during the execution phase and dissemination phase, an advisory panel with user representatives (participants from pilot study, parents of children with pABI and patient organisations) has been appointed and is scheduled to have regular meetings throughout this phase and will be involved in discussions to improve study design, secure a good consent process, relevant assessment instruments and publication in relevant media. Finally, findings to be made available to the participants at the end of study include main findings, where the information is tailored to the characteristics of the sample, in addition to an offer of receiving feedback on individual results.

\section{DISCUSSION}

The proposed study is unique in the field of paediatric cognitive rehabilitation, as it aims to address several limitations of previous studies by applying a robust study design with randomisation to intervention type, large sample size, blinded assessments, involvement of parents and teachers, external cueing and long-term follow-up to obtain solid knowledge on how children and adolescents with $\mathrm{ABI}$ profit from systematic cognitive rehabilitation programme. The study will focus on a vulnerable patient population that has EF impairments that impact multiple domains of everyday life. Hence, it is critical that effective cognitive remediation options are made available for this group of patients and their families. ${ }^{1433} 40 \mathrm{In}$ fact, family-centred interventions have been increasingly recognised as critical in serving children, particularly young children, with disability. ${ }^{68} 69$ The proposed study includes heavy involvement of parents and counselling of teachers (ie, 'context sensitive'), ${ }^{6162}$ which is expected to enhance study adherence and intervention gains. The expected outcome is that pGMT will be more effective in improving EF when compared with $\mathrm{pBHW}$, and that this improvement will generalise to everyday function with potential long-term effects. In the long run, intervention effects may contribute to facilitate completion of education and successful participation in work life and less need of social benefits. It has been suggested that the documented improvements following GMT is a result of underlying alterations in brain networks supporting sustained attention or attentional control. ${ }^{63}$ The capacity to sustain attention over time (eg, attentional control) is a necessity for reliable goal-directed behaviour. So, by targeting sustained attention, which is heavily emphasised in pGMT, improvements in broader domains of goal-directed functioning may occur. As such, we also expect that the intervention will have a positive impact on emotional health, psychosocial health, family function, QoL and perceived fatigue. The study is expected to have a direct impact on rehabilitation practice and may inform rehabilitation programme and guidelines in the paediatric ABI community. As pGMT specifically addresses deficits in sustained attention to improve EF function, the programme may prove helpful to populations from a host of developmental and other acquired aetiologies with $\mathrm{EF}$ challenges.

\section{Author affiliations}

${ }^{1}$ Department of Clinical Neurosciences for Children, Division of Paediatric and Adolescent Medicine, Oslo Universitetssykehus, Oslo, Norway

${ }^{2}$ Children's Clinic, St Olavs's Hospital, Trondheim, Norway

${ }^{3}$ Department of Clinical and Molecular Medicine, Norwegian University of Science and Technology, NTNU, Trondheim, Norway

${ }^{4}$ Department of Public Health and Nursing, Norwegian University of Science and Technology, NTNU, Trondheim, Norway

${ }^{5}$ Department of Psychology, Universitetet i 0slo, Oslo, Norway

${ }^{6}$ Department of Research, Lovisenberg Diaconal Hospital, Oslo, Norway

Acknowledgements The authors thank the participants, patient advisers and the staff at St Olav's Hospital and Oslo University Hospital, Rikshospitalet. We also thank Cathy Catroppa and Brian Levine for discussions concerning the present study. Finally, the study was funded by the Norwegian Research Council.

Contributors REH and AEB are doctoral fellows in the project; they have contributed to the study protocol, and they will also conduct interventions and interpret data. KR, TGF, SA, TBR and JS are senior researchers; they have participated in developing the study protocol and will contribute in interpreting the data. ES, REH and AEB are responsible for the main statistical outcome analysis. KR is the principal investigator. JS translated and adapted GMT to Norwegian. AEB, TGF and JS conceived the original idea for the study design. KR and JS are the main supervisors of the doctoral fellows, with TGF, SA and TBR as cosupervisors. TGF has been mainly responsible for developing the webCRF. REH and AEB contributed equally to this paper. All authors contributed to the final manuscript, including final approval of the version published. Finally, all authors have agreed to be accountable for all aspects of the work.

Funding This work was supported by The Research Council of Norway grant number [260680/H10].

Competing interests None declared.

Patient consent for publication Parental/guardian consent obtained.

Ethics approval The study has been approved by the Regional Committees for Medical and Health Research Ethics, Norway (2017/772/REK) and is currently 
recruiting participants and conducting interventions. The study will be conducted in accordance with the Helsinki declaration and the Ethical Research Involving Children (ChildWatch International and UNICEF).

Provenance and peer review Not commissioned; externally peer reviewed.

Open access This is an open access article distributed in accordance with the Creative Commons Attribution Non Commercial (CC BY-NC 4.0) license, which permits others to distribute, remix, adapt, build upon this work non-commercially, and license their derivative works on different terms, provided the original work is properly cited, appropriate credit is given, any changes made indicated, and the use is non-commercial. See: http://creativecommons.org/licenses/by-nc/4.0/.

\section{REFERENCES}

1. Langlois JA, Rutland-Brown W, Thomas KE. The incidence of traumatic brain injury among children in the United States: differences by race. J Head Trauma Rehabil 2005;20:229-38.

2. Organization WH. World Health Statistics. In: Organization WH. Geneva, 2009.

3. Anderson V, Catroppa C. Advances in postacute rehabilitation after childhood-acquired brain injury: a focus on cognitive, behavioral, and social domains. Am J Phys Med Rehabil 2006;85:767-78.

4. Ryan NP, Anderson V, Godfrey C, et al. Predictors of very-longterm sociocognitive function after pediatric traumatic brain injury: evidence for the vulnerability of the immature "social brain". $J$ Neurotrauma 2014;31:649-57.

5. Ponsford JL, Downing MG, Olver J, et al. Longitudinal follow-up of patients with traumatic brain injury: outcome at two, five, and ten years post-injury. J Neurotrauma 2014;31:64-77.

6. Anderson V, Catroppa C, Morse S, et al. Recovery of intellectual ability following traumatic brain injury in childhood: impact of injury severity and age at injury. Pediatr Neurosurg 2000;32:282-90.

7. Beauchamp M, Anderson V. Cognitive and psychopathological sequelae of pediatric traumatic brain injury. In: Handbook of clinical neurology. Elsevier, 2013: 913-20.

8. Catroppa C, Anderson V. Traumatic brain injury in childhood: rehabilitation considerations. Dev Neurorehabil 2009;12:53-61.

9. Catroppa C, Anderson VA, Muscara F, et al. Educational skills: longterm outcome and predictors following paediatric traumatic brain injury. Neuropsychol Rehabil 2009;19:716-32.

10. Karver CL, Wade SL, Cassedy A, et al. Age at injury and long-term behavior problems after traumatic brain injury in young children. Rehabil Psychol 2012;57:256-65.

11. Li L, Liu J. The effect of pediatric traumatic brain injury on behavioral outcomes: a systematic review. Dev Med Child Neurol 2013;55:37-45.

12. Anderson V, Spencer-Smith M, Wood A. Do children really recover better? neurobehavioural plasticity after early brain insult. Brain 2011;134:2197-221.

13. Klingberg T. Training and plasticity of working memory. Trends Cogn Sci 2010;14:317-24.

14. Ylvisaker M, Feeney T. Pediatric brain injury: social, behavioral, and communication disability. Phys Med Rehabil Clin N Am 2007;18:133-44.

15. Ryan NP, van Bijnen L, Catroppa C, et al. Longitudinal outcome and recovery of social problems after pediatric traumatic brain injury (TBI): contribution of brain insult and family environment. Int J Dev Neurosci 2016;49:23-30.

16. Max JE, Wilde EA, Bigler ED, et al. Psychiatric disorders after pediatric traumatic brain injury: a prospective, longitudinal, controlled study. J Neuropsychiatry Clin Neurosci 2012;24:427-36.

17. Levisohn L, Cronin-Golomb A, Schmahmann JD. Neuropsychological consequences of cerebellar tumour resection in children: cerebellar cognitive affective syndrome in a paediatric population. Brain 2000;123:1041-50.

18. Wilkinson J, Marmol NL, Godfrey C, et al. Fatigue following paediatric acquired brain injury and its impact on functional outcomes: a systematic review. Neuropsychol Rev 2018;28:73-87.

19. Zeltzer LK, Recklitis C, Buchbinder D, et al. Psychological status in childhood cancer survivors: a report from the childhood cancer Survivor study. J Clin Oncol 2009;27:2396-404.

20. Anderson V, Catroppa C. Recovery of executive skills following paediatric traumatic brain injury (TBI): a 2 year follow-up. Brain Inj 2005;19:459-70.

21. Anderson V, Catroppa C, Morse S, et al. Attentional and processing skills following traumatic brain injury in early childhood. Brain Inj 2005;19:699-710.

22. Michaeli O, Kassis I, Shachor-Meyouhas $\mathrm{Y}$, et al. Long-Term motor and cognitive outcome of acute encephalitis. Pediatrics 2014;133:e546-52.
23. Stuss DT. Traumatic brain injury: relation to executive dysfunction and the frontal lobes. Curr Opin Neurol 2011;24:584-9.

24. Stuss DT. Functions of the frontal lobes: relation to executive functions. J Int Neuropsychol Soc 2011;17:759-65.

25. Levine B, Schweizer TA, O'Connor C, et al. Rehabilitation of executive functioning in patients with frontal lobe brain damage with goal management training. Front Hum Neurosci 2011;5:9.

26. Stuss DT, Knight RT. Principles of frontal lobe function. Oxford University Press, 2002.

27. Lewis MW, Babbage DR, Leathem JM. Assessing executive performance during cognitive rehabilitation. Neuropsychol Rehabil 2011:21:145-63.

28. Laatsch L, Harrington D, Hotz G, et al. An evidence-based review of cognitive and behavioral rehabilitation treatment studies in children with acquired brain injury. J Head Trauma Rehabil 2007;22:248-56.

29. Sohlberg MM, Harn B, MacPherson $\mathrm{H}$, et al. A pilot study evaluating attention and strategy training following pediatric traumatic brain injury. Clin Pract Pediatr Psychol 2014;2:263-80.

30. Turner-Stokes L, Williams H, Bill A, et al. Cost-efficiency of specialist inpatient rehabilitation for working-aged adults with complex neurological disabilities: a multicentre cohort analysis of a national clinical data set. BMJ Open 2016;6:e010238.

31. Fountain DM, Burke GAA. Multidisciplinary rehabilitation for children with brain tumors: a systematic review. Dev Neurorehabil 2017;20:68-75

32. Hayes L, Shaw S, Pearce MS, et al. Requirements for and current provision of rehabilitation services for children after severe acquired brain injury in the UK: a population-based study. Arch Dis Child 2017; 102:813-20.

33. Limond J, Leeke R. Practitioner review: cognitive rehabilitation for children with acquired brain injury. J Child Psychol Psychiatry 2005;46:339-52.

34. Robinson KE, Kaizar E, Catroppa C, et al. Systematic review and meta-analysis of cognitive interventions for children with central nervous system disorders and neurodevelopmental disorders. $J$ Pediatr Psychol 2014;39:846-65.

35. Butler RW, Copeland DR, Fairclough DL, et al. A multicenter, randomized clinical trial of a cognitive remediation program for childhood survivors of a pediatric malignancy. J Consult Clin Psychol 2008;76:367-78.

36. Galbiati S, Recla M, Pastore V, et al. Attention remediation following traumatic brain injury in childhood and adolescence. Neuropsychology 2009;23:40-9.

37. Luton LM, Reed-Knight B, Loiselle K, et al. A pilot study evaluating an abbreviated version of the cognitive remediation programme for youth with neurocognitive deficits. Brain Inj 2011;25:409-15.

38. Sjö NM, Spellerberg S, Weidner S, et al. Training of attention and memory deficits in children with acquired brain injury. Acta Paediatr 2010;99:230-6.

39. van 't Hooft I, Andersson K, Bergman B, et al. Sustained favorable effects of cognitive training in children with acquired brain injuries. NeuroRehabilitation 2007;22:109-16.

40. Lindsay S, Hartman LR, Reed N, et al. A systematic review of Hospital-to-School reintegration interventions for children and youth with acquired brain injury. PLoS One 2015;10:e0124679.

41. Cicerone KD, Langenbahn DM, Braden C, et al. Evidence-Based cognitive rehabilitation: updated review of the literature from 2003 through 2008. Arch Phys Med Rehabil 2011;92:519-30.

42. Kennedy MRT, Coelho C, Turkstra L, et al. Intervention for executive functions after traumatic brain injury: a systematic review, metaanalysis and clinical recommendations. Neuropsychol Rehabil 2008;18:257-99.

43. Levine B, Robertson IH, Clare L, et al. Rehabilitation of executive functioning: an experimental-clinical validation of goal management training. J Int Neuropsychol Soc 2000;6:299-312.

44. Spikman JM, Boelen DHE, Lamberts KF, et al. Effects of a multifaceted treatment program for executive dysfunction after acquired brain injury on indications of executive functioning in daily life. J Int Neuropsychol Soc 2010;16:118-29.

45. Stamenova V, Levine B. Effectiveness of goal management training ${ }^{\circledR}$ in improving executive functions: a meta-analysis. Neuropsychol Rehabil 2018:1-31.

46. Stubberud J, Langenbahn D, Levine B, et al. Goal management training of executive functions in patients with spina bifida: a randomized controlled trial. J Int Neuropsychol Soc 2013;19:672-85.

47. Tornås S, Løvstad M, Solbakk A-K, et al. Rehabilitation of executive functions in patients with chronic acquired brain injury with goal management training, external Cuing, and emotional regulation: a randomized controlled trial. J Int Neuropsychol Soc 2016;22:436-52.

48. Krasny-Pacini A, Chevignard M, Evans J. Goal management training for rehabilitation of executive functions: a systematic review of 
effectiveness in patients with acquired brain injury. Disabil Rehabil 2014;36:105-16.

49. Metzler-Baddeley C, Jones RW. Brief communication: cognitive rehabilitation of executive functioning in a case of craniopharyngioma. Appl Neuropsychol 2010;17:299-304.

50. Novakovic-Agopian T, Chen AJ-W, Rome S, et al. Rehabilitation of executive functioning with training in attention regulation applied to individually defined goals: a pilot study bridging theory, assessment, and treatment. J Head Trauma Rehabil 2011;26:325-38.

51. Schweizer TA, Levine B, Rewilak D, et al. Rehabilitation of executive functioning after focal damage to the cerebellum. Neurorehabil Neural Repair 2008;22:72-7.

52. Miotto EC, Evans JJ, Souza de Lucia MC, et al. Rehabilitation of executive dysfunction: a controlled trial of an attention and problem solving treatment group. Neuropsychol Rehabil 2009;19:517-40.

53. Stubberud J, Langenbahn D, Levine B, et al. Goal management training improves everyday executive functioning for persons with spina bifida: self-and informant reports six months post-training. Neuropsychol Rehabil 2014;24:26-60.

54. Stubberud J, Langenbahn D, Levine B, et al. Emotional health and coping in spina bifida after goal management training: a randomized controlled trial. Rehabil Psychol 2015;60:1-16.

55. Tornås S, Løvstad M, Solbakk A-K, et al. Goal management training combined with external cuing as a means to improve emotional regulation, psychological functioning, and quality of life in patients with acquired brain injury: a randomized controlled trial. Arch Phys Med Rehabil 2016;97:1841-52.

56. Robertson IH, Garavan H. Vigilant attention. In: Gazzaniga MS ed. The cognitive neurosciences. Cambridge, MA: MIT Press, 2004: 631-40.

57. Chen AJ-W, Novakovic-Agopian T, Nycum TJ, et al. Training of goaldirected attention regulation enhances control over neural processing for individuals with brain injury. Brain 2011;134:1541-54.

58. Stuss DT, Knight RT. Principles of frontal lobe function. Oxford University Press, 2013.

59. Kabat-Zinn J. Full catastrophe living. New York: Dell Publishing, 1990.

60. Krasny-Pacini A, Limond J, Evans J, et al. Context-Sensitive goal management training for everyday executive dysfunction in children after severe traumatic brain injury. J Head Trauma Rehabil 2014;29:E49-64.

61. Braga LW, Rossi L, Moretto ALL, et al. Empowering preadolescents with $A B I$ through metacognition: preliminary results of a randomized clinical trial. NeuroRehabilitation 2012;30:205-12.

62. Ylvisaker M, Adelson PD, Braga LW, et al. Rehabilitation and ongoing support after pediatric TBI: twenty years of progress. $J$ Head Trauma Rehabil 2005;20:95-109.

63. Stubberud J, Holthe I, Løvstad M, et al. Goal management training of executive functions inchildren with spina bifida and acquired brain injury: a pilot study. Neuropsychological Rehabilitation 2019. Under review.

64. Organization WH. Neurological disorders: public health challenges. World Health Organization, 2006.

65. Anderson VA, Anderson P, Northam E, et al. Relationships between cognitive and behavioral measures of executive function in children with brain disease. Child Neuropsychol 2002;8:231-40.

66. Gioia GA, Isquith PK, Guy SC, et al. Behavior rating inventory of executive function: professional manual. Lutz, FL: Psychological Assessment Resources, Inc, 2000.

67. Schulz KF, Altman DG, Moher D. Consort 2010 statement: updated guidelines for reporting parallel group randomised trials. BMC Med 2010;8:18.

68. Baber N. International Conference on harmonisation of technical requirements for registration of pharmaceuticals for human use (ICH). Br J Clin Pharmacol 1994;37:401-4.

69. Gjærum B. Hjerne og atferd: utviklingsforstyrrelser hos barn og ungdom i et nevrobiologisk perspektiv:-et skritt videre. Gyldendal Akademisk, 2002.

70. Helseth E, Due-Tønnessen B, Lundar T, et al. Intracranial tumors in children. Tidsskrift for den Norske laegeforening: tidsskrift for praktisk medicin, ny raekke 2003;123.
71. Mallick AA, O'Callaghan FJK. The epidemiology of childhood stroke. Eur J Paediatr Neurol 2010;14:197-205.

72. HH. Acute viral encephalitis in children: Pathogenesis, incidence, and etiology. In: Morven MS, Armsby C, eds. UpToDate, 2018.

73. Chan DYK, Fong KNK. The effects of problem-solving skills training based on metacognitive principles for children with acquired brain injury attending mainstream schools: a controlled clinical trial. Disabil Rehabil 2011;33:2023-32.

74. Wechsler D. Wechsler intelligence scale for children. 5th edn. TX, San Antonio: Psychological Corporation, 2014.

75. Sørensen LH M. Psychometric properties of the Norwegian version of behavior rating inventory of executive function, parent version (BRIEF-P). PsykTestBarn 2014;2.

76. Chevignard MP, Catroppa C, Galvin J, et al. Development and evaluation of an ecological task to assess executive functioning post childhood TBI: the children's cooking task. Brain Impairment 2010;11:125-43.

77. Chevignard MP, Servant V, Mariller A, et al. Assessment of executive functioning in children after TBI with a naturalistic open-ended task: a pilot study. Dev Neurorehabil 2009;12:76-91.

78. Fish J, Evans JJ, Nimmo M, et al. Rehabilitation of executive dysfunction following brain injury: "content-free" cueing improves everyday prospective memory performance. Neuropsychologia 2007:45:1318-30.

79. Manly T, Hawkins K, Evans J, et al. Rehabilitation of executive function: facilitation of effective goal management on complex tasks using periodic auditory alerts. Neuropsychologia 2002;40:271-81.

80. Emslie H, Burden V, Nimmo-Smith I, et al. Behavioural assessment of the dysexecutive syndrome for children: Thames Valley test company 2003.

81. DuPaul GJ, Power TJ, Anastopoulos AD, et al. Adhd rating ScaleIV (for children and adolescents): checklists, norms, and clinical interpretation. Guilford Publications, 1998.

82. Conners C. Conners Continuous Performance Test 3rd edition ${ }^{\mathrm{TM}}$ (Conners CPT $3^{\mathrm{TM}}$ ), Conners Continuous Auditory Test of Attention (Conners CATA ${ }^{\mathrm{TM}}$ ) manual. Toronto: Multi-Health Systems Inc, 2014.

83. Delis DC, Kaplan E, Kramer JH. Delis-Kaplan executive function system: examiners manual. Psychological Corporation, 2001.

84. Culbertson W, Zillmer E. Tower of London: Examiner's manual. North Towanda, NY: Multi" Health Systems, 1999

85. Kløve H. Clinical neuropsychology. Med Clin North Am 1963;47:1647-58.

86. Delis DC. California verbal learning test. Adult version Manual Psychological Corporation, 2000.

87. Achenbach TM. Child behavior checklist for ages 6-18. Burlington: ASEBA. University of Vermont, 2001.

88. Korkman M, Kirk U, Kemp S. NEPSY. 2nd edn. San Antonio: TX: Psychological Corporation, 2007.

89. Harter S. Manual for the self-perception profile for children:(revision of the perceived competence scale for children). University of Denver, 1985.

90. Varni JW, Seid M, Rode CA. The PedsQL: measurement model for the pediatric quality of life inventory. Med Care 1999;37:126-39.

91. Ayr LK, Yeates KO, Taylor HG, et al. Dimensions of postconcussive symptoms in children with mild traumatic brain injuries. $J$ Int Neuropsychol Soc 2009;15:19-30.

92. Noller P, Seth-Smith M, Bouma R, et al. Parent and adolescent perceptions of family functioning: a comparison of clinic and nonclinic families. J Adolesc 1992;15:101-14.

93. Radloff LS. The CES-D scale: a self-report depression scale for research in the general population. Appl Psychol Meas 1977;1:385-401.

94. Beers SR, Wisniewski SR, Garcia-Filion P, et al. Validity of a pediatric version of the Glasgow outcome Scale-Extended. J Neurotrauma 2012;29:1126-39.

95. Wille N, Badia X, Bonsel G, et al. Development of the EQ-5D-Y: a child-friendly version of the EQ-5D. Qual Life Res 2010;19:875-86. 
Correction: paediatric goal management training in patients with acquired brain injury: study protocol for a randomised controlled trial

Hypher RE, Brandt AE, Risnes K, et al. Paediatric goal management training in patients with acquired brain injury: study protocol for a randomised controlled trial. BMJ Open 2019;9:e029273. doi: 10.1136/bmjopen-2019-029273

The previous version of this manuscript left out some information regarding Co-authorship.

Authors, Ruth Elizabeth Hypher and Anne Elisabeth Brandt are joint first authors and contributed equally to this paper.

Open access This is an open access article distributed in accordance with the Creative Commons Attribution Non Commercial (CC BY-NC 4.0) license, which permits others to distribute, remix, adapt, build upon this work non-commercially, and license their derivative works on different terms, provided the original work is properly cited, appropriate credit is given, any changes made indicated, and the use is non-commercial. See:http://creativecommons.org/ licenses/by-nc/4.0/

C Author(s) (or their employer(s)) 2019. Re-use permitted under CC BY-NC. No commercial re-use. See rights and permissions. Published by BMJ.

BMJ Open 2019;9:e029273corr1. doi:10.1136/bmjopen-2019-029273corr1

Check for updates 